\title{
The challenges of Proteus syndrome: diagnosis and management
}

Proteus syndrome (PS) is a disorder of patchy or mosaic postnatal overgrowth of unknown etiology. The onset of overgrowth typically occurs in infancy and can involve any tissue of the body. Commonly involved tissues include connective tissue and bone, skin, central nervous system, the eye, but it apparently can affect any tissue. Diagnosing of PS is difficult and the diagnostic criteria are controversial. Our group advocates stringent diagnostic criteria to facilitate research and appropriate clinical care. The benefit of strict criteria is that they define a clinical group that is reasonably homogenous with respect to manifestations and prognosis. The overgrowth of PS is progressive and can be difficult to manage. The progressive overgrowth most commonly causes severe orthopaedic complications, but it can cause many other complications. One of the most common complications in patients with PS is deep venous thrombosis and pulmonary embolism, which can cause premature death. Effective management requires knowledge of the wide array of manifestations and complications of the disorder and a team approach that includes the geneticist, surgeons, and other specialists.

\section{In brief}

- Proteus syndrome (PS) is an extremely rare and overdiagnosed disorder of mosaic growth dysregulation, primarily involving overgrowth.

- The disorder is thought to be caused by a somatic genetic alteration but the etiology is unknown.

- Clinical diagnosis of PS is challenging and controversial.
- The management of PS is also challenging, primarily owing to the aggressive and disproportionate postnatal overgrowth.

- Patients with PS have an increased risk of premature death, commonly caused by deep venous thrombosis and pulmonary embolism.

- Patients with PS have an increased risk of developing tumors.

\section{Introduction}

Proteus syndrome (PS) was described as a discrete clinical entity in $1979^{1}$ and assigned its name several years later. ${ }^{2}$ This is a relatively recently delineated syndrome, probably

\footnotetext{
Leslie Biesecker*,

${ }^{1}$ National Human Genome Research Institute, Building 49 Room 4A80, Bethesda, MD 20892, USA
}

European Journal of Human Genetics (2006) 14, 1151-1157. doi:10.1038/sj.ejhg.5201638; published online 2 August 2006

Keywords: overgrowth; mosaicism; macrodactyly; cancer predisposition; thrombophilia

${ }^{*}$ Correspondence: Dr L Biesecker, National Human Genome Research Institute, Building 49 Room 4A80, Bethesda, MD 20892, USA. Tel: +1301 402 2041; Fax: + 1301402 2170;

E-mail: leslieb@helix.nih.gov

This article is in the public domain in the United States of America.

Received 23 December 2005; revised 6 February 2006; accepted 8 March 2006; published online 2 August 2006 because it is so rare and because it overlaps with a number of other asymmetric overgrowth syndromes. Of course, the syndrome is not 'new' and the oldest known case is that of Mr Joseph Merrick, described by Treves in the 19th century., ${ }^{3,4}$ The rarity of PS contributes to confusion and controversy regarding the disorder, especially regarding the diagnosis. We advocate for stringent diagnostic criteria, which delineate a distinct cohort of patients who have a similar type of overgrowth and a predictable set of complications..$^{5-7}$ However, this does not imply that all patients with PS have a similar degree of severity. In fact, PS causes a remarkable degree of interpatient variation in the severity and the extent of the involvement. A review of reported cases that were re-screened using the proposed diagnostic criteria suggests that important insights can be gained by studying well-defined cohorts. ${ }^{8}$ The present review first delineates the manifestations of the disorder and then considers the published diagnostic criteria. Finally, some issues of management, prognosis and etiology are considered. 


\section{Manifestations}

The disorder primarily manifests as postnatal overgrowth, with irregular, distorting and progressive overgrowth that can include many tissues. All patients with PS manifest the phenotype in a patchy or mosaic pattern. The manifestations are typically asymmetric and the location of the manifestations varies remarkably among the patients. Some patients have a strikingly limited extent of the manifestations, which can make diagnosis challenging, as described below. The manifestations of PS apparently can include any tissue of the body. Although bone, connective tissue and fat are the most commonly involved tissues, we have evaluated patients with overgrowth of the central nervous system, spleen, thymus, colon and other tissues. The manifestations of overgrowth are almost always asymmetric. In fact, the presentation of overgrowth in a symmetric pattern (eg, overgrowth of both hands and both feet) should lead one to doubt the diagnosis of PS, as this is rare in confirmed cases. The second general feature of PS is that it is rapidly progressive and typically occurs at a frighteningly rapid pace. Most patients with PS are born without significant asymmetry and the asymmetric overgrowth starts in the 6-18 months age range. The rate of overgrowth is such that affected body parts rapidly become disproportionate. The nature of the overgrowth in PS is also distinctive, when compared to other disorders that manifest asymmetric overgrowth. It is termed irregular and progressive to distinguish it from the more common form of overgrowth, which is proportionate and regular or what we have termed 'ballooning' (Figure 1). In patients with PS, the overgrowth typically affects the bone underlying the enlarged body part in a remarkably irregular fashion causing the body part and the underlying bone to lose its normal architecture. Indeed, one of the hallmarks of the disorder is that the affected bones can become unrecognizably distorted. Although it is the limbs that are most commonly involved, any body part can overgrow.

Vascular malformations are also common in PS. ${ }^{9,10}$ These are most commonly cutaneous capillary malformations, but some patients have venous malformations and we are aware of one unambiguously affected patient who has intracranial arteriovenous malformations. Overall, we believe that arterial vascular malformations are uncommon in PS. It is worth noting that the vascular malformations of PS are not typically as severe as are those in the Klippel-Trenaunay syndrome ${ }^{11}$ (KTS). As well, PS can be distinguished from KTS because it has a number of specific manifestations that are not seen in KTS such as cerebriform connective tissue nevi.

Dysregulation of fatty tissue is a hallmark of PS. In fact, one of the more intriguing manifestations of PS is that it can include both overgrowth and atrophy of fatty tissues. Although many patients are described as having 'lipomas', it should be recognized that these are actually localized overgrowth of fatty tissue in areas that normally have such tissue and these fatty tissue overgrowths are not encapsulated, as are the common, ovoid lipomas seen in elderly patients. Many patients with confirmed PS have had significant, and even severe localized overgrowth of fatty tissue in the abdomen or extremities and also had lipoatrophy in other areas of the body, most commonly the chest. For this reason, we refer to PS as manifesting
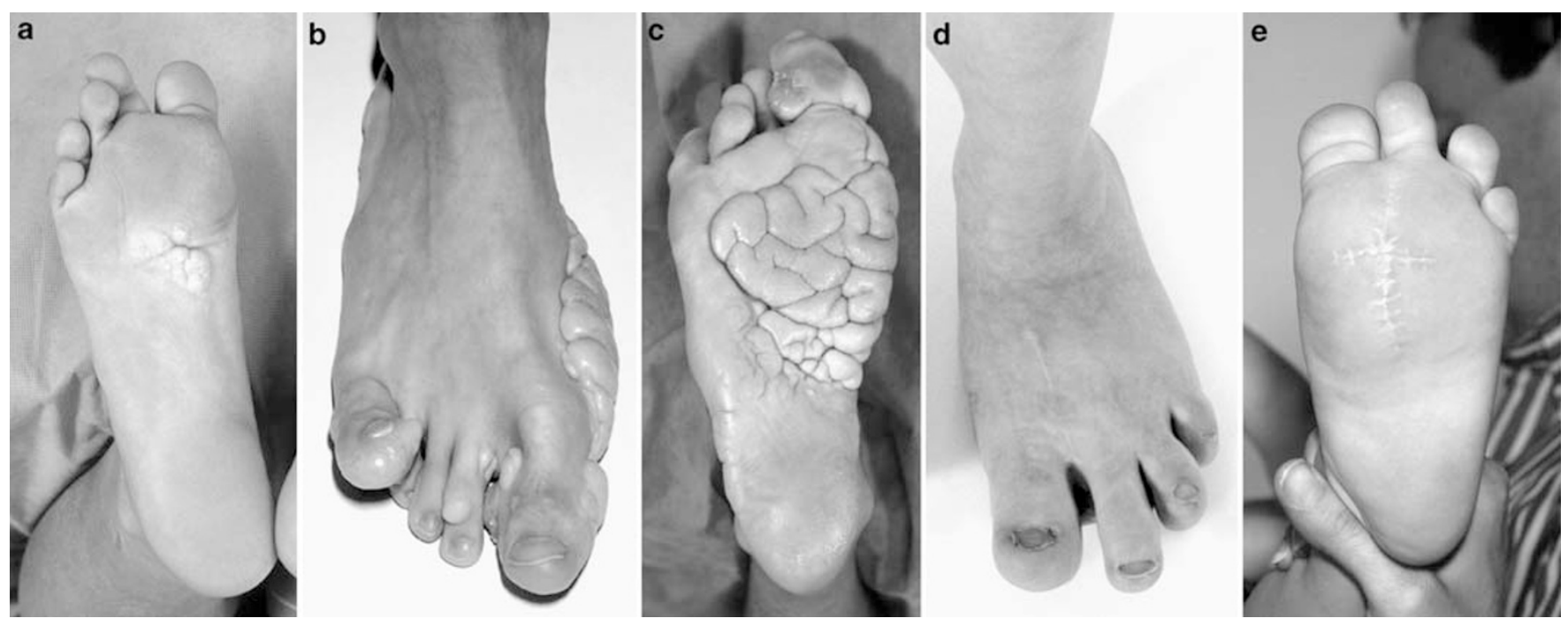

Figure 1 The distinction of overgrowth in a patient with PS (a-c) from that of a patient with hemihyperplasia (d, e). Panel a shows an early CCTN. Note that the appearance of this lesion is distinct from that of wrinkling of normal sole tissue. Panels $\mathbf{b}$ and $\mathbf{c}$ are from a patient with more advanced PS demonstrates several features of the disorder. Panel $\mathbf{b}$ shows distorting overgrowth of the first and fifth digits (arrows) with loss of the normal architecture of the digits. The overgrowth in the patient with PS is associated with calcified tissue around the epiphyses that restricts movement at the affected joints. The overgrowth in the patient with hemihyperplasia $(\mathbf{d}, \mathbf{e})$ is described by as 'ballooning' overgrowth as it is not associated with distortion. 
dysregulated fatty tissues instead of simply describing it as manifesting lipomas.

Another common skin manifestation in PS is the linear verrucous epidermal nevus. ${ }^{9,10}$ This lesion may be present at birth in some patients, and if it is congenital, it is typically subtle and may be macular at that time. In most patients, it is not present at birth and instead the skin begins to thicken and darken in the first year of life. The lesion is typically dark brown to almost black, rough in texture, patchy and can follow the lines of Blaschko. A less common, but fairly specific skin lesion in PS is the cerebriform connective tissue nevus (CCTN). We have never observed a patient who was said to have a CCTN manifest at birth. The lesion evolves slowly, in some patients developing through adolescence. It begins as a localized thickening of the subcutaneous tissue and with time develops marked thickening, which may be a centimeter or more deep. This thickening commonly alternates with deep grooves, lending the lesion its 'cerebriform' descriptor. The lesion is found most commonly on the sole of the feet, less commonly on the hands and occasionally at the nares or medial puncta of the eyes. One patient had an extensive CCTN present in a shield shaped distribution on the chest wall. We suspect that this lesion can occur anywhere on the body but that it is rare in places other than those mentioned here.

The facial phenotype of PS is present in a minority of patients, more commonly in those with cognitive deficits (for unknown reasons). This phenotype comprises downslanting palpebral fissures, flattening of the malar bones, relative lengthening of the face and a persistently open mouth.

PS appears to be associated with a number of tumors. It is difficult to be certain about this association for two reasons. First, PS is very rare and there is almost certainly ascertainment bias regarding the reporting of cases with tumors. Second, the associated tumors are of widely varying types. The two types of tumors most specifically associated with PS are monomorphic adenomas of the parotid glands and bilateral ovarian cystadenomas. These tumors are used in the diagnostic criteria because of their specificity. A wide variety of other tumors have been reported in patients with PS including unilateral ovarian cystadenomas and meningiomas (for a review, see Cohen et $\mathrm{al}^{12}$ ), but none of these tumors are sufficiently specific to warrant being considered as a diagnostic sign.

\section{Diagnosis}

As mentioned above, there is controversy regarding the diagnosis of PS. Although it is clear that the patients described by Wiedemann et $a l^{2}$ and Cohen and Hayden ${ }^{1}$ had a distinct and unitary clinical entity, many of the subsequently described patients have substantially diver- gent manifestations compared to these original patients. There are a number of reasons for this phenomenon. Early descriptions of PS were not accompanied by defined diagnostic criteria. Although in retrospect it is clear that those early reports included patients whose manifestations were recognizably distinct from those of many other overgrowth entities, this must not have been apparent to other observers at the time. It is also clear that there are likely to be a substantial number of discrete clinical entities that include asymmetric overgrowth as a manifestation, as discussed by Wiedemann and Burgio ${ }^{13}$ almost 20 years ago. As has been true for many pleiotropic disorders described over the past 30 years, it can take some time for clinical researchers and practitioners to recognize these distinctions. Although it is now obvious to most observers, it must be stated that not all patients with asymmetric overgrowth, vascular malformations and skin lesions have PS. Our experience in researching PS suggests that the triad of findings (asymmetric overgrowth, vascular anomalies and skin manifestations) probably encompasses 5-10 distinct disorders in addition to PS including NF1, hemihyperplasia and multiple lipomatosis syndrome (HHML) and a number of provisionally unique syndromes (Cohen and Biesecker, unpublished observations).

To address this issue, diagnostic criteria were delineated. ${ }^{6}$ Again, these criteria are controversial so it is appropriate to review the history and rationale for their generation. Previous attempts have been made to develop criteria, although these have been supplanted by more recent efforts. One set of obsolete criteria were developed ${ }^{14}$ that were later modified by another group. ${ }^{15}$ These criteria are clearly inadequate as they allow the label of PS to be applied to patients who clearly have hemihyperplasia, as exemplified by the case described in the report with the modified criteria. ${ }^{15}$ The more recent criteria emanated from our experience with a large clinic where we evaluated 18 patients who had a diagnosis of PS. ${ }^{7}$ The ability to see these many patients simultaneously was a unique experience, again as PS is very rare. At this clinic, it was immediately and obviously apparent (to the physicians, counselors and parents) that it was not possible that all the patients had the same condition. The families and we recognized that some of the patients had a static and generally milder condition that did not include CCTN, progressive overgrowth or the facial features described above. By evaluating a large cohort simultaneously, it was easy to divide the patients into three groups; the first group had the same condition described by Wiedemann ${ }^{2}$ and Cohen $^{1}$ (eight patients, labeled as PS), the second group had a distinct phenotype that appeared to be a form of hemihyperplasia (eight patients, diagnosed with the newly designated HHML syndrome) and the final group had two patients, each of whom appeared to have their own, distinct disorder of overgrowth. ${ }^{7}$ This experience was reviewed and formalized in a workshop that took place at 
Table 1 Revised PS diagnostic criteria.

\begin{tabular}{|c|c|}
\hline \multicolumn{2}{|c|}{ To make a diagnosis of PS, one must have all the general criteria, and various specific criteria } \\
\hline $\begin{array}{l}\text { General Criteria } \\
\text { All the following: } \\
\text { Mosaic distribution of lesions } \\
\text { Sporadic occurrence } \\
\text { Progressive course }\end{array}$ & $\begin{array}{l}\text { Specific Criteria } \\
\text { Either: } \\
\text { Category A or, } \\
\text { Two from category B or, } \\
\text { Three from category C }\end{array}$ \\
\hline $\begin{array}{l}\text { Specific criteria categories } \\
\text { A. } \\
\text { 1. Cerebriform connective tissue nevus } \\
\text { 1. Linear epidermal nevus } \\
\text { 2. Asymmetric, disproportionate overgrowth } \\
\text { One or more: } \\
\text { (a) Limbs } \\
\text { (b) Hyperostosis of the skull } \\
\text { (c) Hyperostosis of the external auditory canal } \\
\text { (d) Megaspondylodysplasia } \\
\text { (e) Viscera: } \\
\text { Spleen/thymus } \\
\text { 3. Specific tumors before 2nd decade } \\
\text { One of the following: } \\
\text { (a) Bilateral ovarian cystadenoma } \\
\text { (b) Parotid monomorphic adenoma }\end{array}$ & $\begin{array}{l}\text { 1. Dysregulated adipose tissue } \\
\text { Either one: } \\
\text { (a) Lipomas } \\
\text { (b) Regional lipohypoplasia } \\
\text { 2. Vascular malformations } \\
\text { One or more: } \\
\text { (a) Capillary malformation } \\
\text { (b) Venous malformation } \\
\text { (c) Lymphatic malformation } \\
\text { 3. Lung cysts } \\
\text { 4. Facial phenotype } \\
\text { All: } \\
\text { (a) Dolichocephaly } \\
\text { (b) Long face } \\
\text { (c) Down slanting palpebral fissures and/or minor ptosis } \\
\text { (d) Low nasal bridge } \\
\text { (e) Wide or anteverted nares } \\
\text { (f) Open mouth at rest }\end{array}$ \\
\hline
\end{tabular}

This table is adapted from prior publications. ${ }^{6,8,21}$

${ }^{a}$ Cerebriform connective tissue nevi are skin lesions characterized by deep grooves and gyrations as seen on the surface of the brain. See text and Figure 1 for details.

${ }^{\mathrm{b}}$ Asymmetric, disproportionate overgrowth should be carefully distinguished from asymmetric, proportionate, or ballooning overgrowth. See text and Figure 1 for details.

the NIH campus in 1998, concurrently with a meeting of the United States Proteus syndrome support group. The meeting conferees reviewed the data on a large number of patients (published and unpublished) and distilled those data into a set of diagnostic criteria. ${ }^{6}$

The diagnostic criteria have two categories of attributes; general and specific. The general attributes delineate the nonspecific features of PS by requiring that all patients have a mosaic distribution of the phenotype, are sporadic and the manifestations are progressive in nature. If a patient does not have all three of these criteria, the diagnosis of PS is rejected. If a patient has all three of the general attributes, the specific criteria should be assessed. These are listed in Table 1. Some comments are necessary regarding several of these criteria. First, the CCTN (specific criterion A) is not equivalent to some wrinkling of the skin owing to subcutaneous overgrowth or lipomas. In fact, the CCTN involves a marked thickening of the cutaneous and subcutaneous tissues, typically with whorls of collagencontaining cells. Second, disproportionate overgrowth (specific criterion B2) does not include lipomas. It is well known that lipomas can expand with age and this expansion does not count for this criterion (instead it counts for specific criterion C1). As well, the disproportionate overgrowth that does occur is nearly always postnatal, severe, rapid and relentless. The affected tissues grow much faster than the unaffected tissues and the patients become markedly more deformed with time. If the clinician is in doubt about whether the patient has this manifestation, the criterion is not met.

We have found these criteria relatively easy to use, but some others have not. Although our internal consistency is high (199/205 cases yielded congruent assessment by three observers; MM Cohen Jr., J Turner and this author), there have been a number of cases where we did not agree with the application of these diagnostic criteria by others (eg, see Beisecker et $a l^{16}$, Smith et al ${ }^{17}$ and Zhou et al ${ }^{18}$. Many cases where we disagree with the claim that a patient has PS are based on reports that include inadequate clinical data.

The diagnostic criteria have utility in two arenas: clinical and research. In the clinic, it is abundantly clear that patients who meet the diagnostic criteria will follow a progressive course. This can be very discouraging for families, but it is helpful for clinicians to be aware of the issue. As noted above, patients with true PS generally suffer severe and relentless overgrowth. We have evaluated patients who experienced differential leg growth such that they had more than $20 \mathrm{~cm}$ leg length difference occuring in just a few years, whereas little or no asymmetry was present at birth. Another patient had the postnatal onset of hyperostosis of the skull, which transformed from normal to a $4-\mathrm{cm}$ thick skull table in just a few years. Although 
these are not meant to represent the average growth rate in patients with PS, they should give the reader a clear picture that the overgrowth is severe. As was said above, if one is unsure if the patient has progressive overgrowth, they do not. In addition, the patients who meet the criteria have several other distinct attributes. The rate of premature death in published patients with true PS is $20 \%$, compared to $4 \%$ for patients who are claimed to have PS but do not meet the criteria. ${ }^{8}$ Surprisingly, the ratio of males to females among reported cases who meet the diagnostic criteria is $1.9: 1 .^{8}$ As there is little reason to suspect a priori that the diagnostic criteria would select for males or for lethality, these results suggest that the diagnostic criteria do identify a distinct biological group. It is acknowledged that one should avoid the circular reasoning of validating strict criteria because the resulting cohort has uniform attributes. However, we have been impressed that the diagnostic criteria identify significant associations with attributes not included in the criteria. ${ }^{8}$ The great majority of patients who are referred to the NIH study but do not meet the criteria appear to have the HHML phenotype. In those patients, the prognosis is good as they do not appear to suffer from the aggressive overgrowth of PS and do not appear to be as susceptible to thrombosis (see below). However, patients with HHML are likely to be at elevated risk for Wilm's tumor and possibly hepatoblastoma, so it is appropriate to consider following the standard screening protocols for those tumors. ${ }^{19}$

The diagnostic criteria are not without controversy. First, the literature has more reports of patients misdiagnosed than correctly diagnosed. ${ }^{8}$ Some seem to argue that essentially all asymmetric overgrowth with vascular anomalies, lipomas or dermatologic symptoms is part of a broad and continuous spectrum that subsumes PS, KTS and various forms of syndromic hemihyperplasia (eg, HHML). Patients with KTS, PS and HHML do not follow similar courses and the clinician would be foolish to believe that they do. The second controversy surrounds the issue of PTEN mutations and PS. It has been claimed that several patients with PS or 'Proteus-like syndrome' have mutations in PTEN. ${ }^{17,18,20}$ This argument will not be repeated here, but suffice it to say that most of these case reports include insufficient clinical data to make a diagnosis and in the few that do include sufficient clinical data, it is clear that the patients do not meet the PS diagnostic criteria. ${ }^{8,16,21-23}$ As well, several groups have tested a large number of patients with typical PS and found no mutations in this gene. ${ }^{16,24}$ As we have said before, the criteria may need to be adapted from time to time, as they have been in the past. ${ }^{8}$ Ideally, such adaptations should reflect an evolving understanding of the phenotype in the context of developing knowledge regarding etiology. ${ }^{25}$

Note should also be made that there are a number of patients with very mild PS. Typically, such patients are mild because the portion of their body that is affected is small, not because they have substantial areas affected with mild disease. This can make diagnosis challenging and it is acknowledged that there may be patients whose delimited phenotype is not captured by the diagnostic criteria. Again, it should be emphasized that it is the quantity of affected tissue that determines the severity of the syndrome but it is the nature of the symptoms that determines whether the patient has PS or an overlapping overgrowth disorder other than PS. Nearly all misdiagnosed patients are misdiagnosed because the nature of their signs was mis-assessed, not because of the extent or severity of the signs.

\section{Management}

The management of patients with PS is challenging. ${ }^{26}$ The rapid overgrowth causes severe problems in most patients. We have found it important, but difficult to manage the overgrowth in patients with PS. The treatment of overgrowth typically involves multiple orthopedic procedures over years to decades to control the overgrowth. In spite of aggressive treatment, patients can suffer severe functional and cosmetic consequences of this disorder (Figure 2). We have noted a paradox in caring for patients with PS. It is often said that it is best to delay surgical intervention until it is clear that it is 'absolutely necessary'. However, when it is 'absolutely necessary', the severity of the complication is such that the patient is deemed to be a poor surgical candidate and that surgery is contraindicated. We have been caught in this paradox ourselves and it is terribly frustrating. In such cases, one may need to proceed with surgery before the complication becomes debilitating.

It is now clear that one of the most common causes of death for patients with PS is deep venous thrombosis and pulmonary embolism, even in young children. ${ }^{27,28}$ Nearly all patients with PS who have been operated on at the NIH for complications related to PS have experienced some degree of difficulty with thrombosis. For this reason, we strongly recommend that all patients be considered for anticoagulant prophylaxis perioperatively. This prophylaxis can be difficult to implement because patients with PS may have vascular anomalies and the surgeries may be complicated by postoperative bleeding (eg, spinal fusions). Nevertheless, there are a number of unpublished cases known to this author of perioperative death from thrombosis and or embolism in patients with PS. For this reason, we routinely use anticoagulant prophylaxis and monitor patients closely for thromboses. In contrast to this strong recommendation to perform perioperative anticoagulant prophylaxis, we do not recommend chronic anticoagulation. There are no data to assess the risks and benefits of this approach and the long-term risks may be high in the setting of a syndrome with a high frequency of vascular anomalies. 

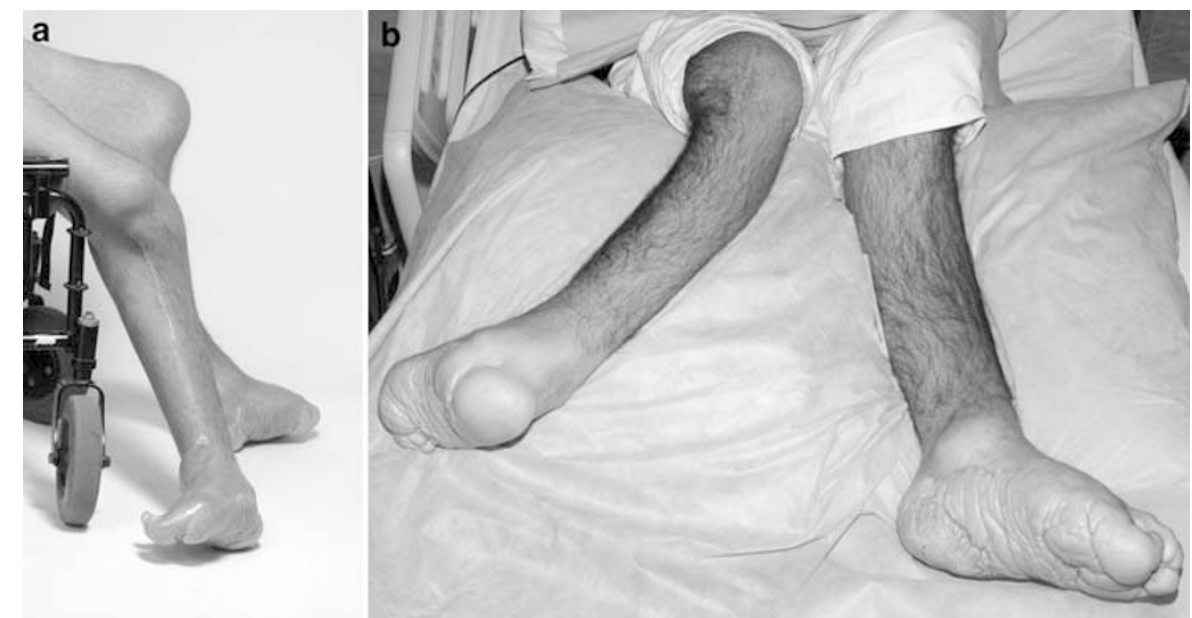

Figure 2 The progressive nature and severity of overgrowth in PS. Panel a shows a teenager with valgus deformity of the knee, fixation of the knee joint due to bony overgrowth surrounding the joint and external rotation of lower leg of more than $90^{\circ}$. Panel $\mathrm{b}$ shows the same patient a few years later with continued external rotation of the leg that now exceeds $135^{\circ}$ with onset of bowing of the tibia. The surgical correction of such deformities is challenging.

The aforementioned predisposition to tumors is a thorny problem. First, the association is strongly suspected, but not proven. Second, although there are a number of tumor types that have occurred in more than one patient with $\mathrm{PS}^{29}$ the predisposition appears to be broad (ie, predisposition to a wide variety of tumors) and are not aware of any practical and effective method to screen for such tumors. Finally, there are no data to demonstrate that early detection of tumors in PS improves prognosis. For these reasons, we do not recommend routine imaging surveillance for tumors. Instead, we recommend regular check ups with a physician who has a relatively low threshold for ordering an appropriate evaluation if a patient develops signs or symptoms of a tumor.

\section{Pathogenetic model and future research}

The currently accepted pathogenetic model was proposed by Happle. ${ }^{30,31}$ He suggested that PS was caused by a somatic alteration (eg, mutation), that the tissues derived from that mutant clone manifested the symptoms of PS, and that the mutation is embryonic lethal in the nonmosaic state. This would explain a number of attributes of PS including the lack of recurrence in sibs or in offspring of affected patients, the existence of discordant monozygotic twins and the absence of diffusely affected patients (ie, all are patchy and the affected tissues vary widely among the patients). Again, this is a model and there are no data that prove this is correct. On the basis of this model and clinical experience, we give patients a very low recurrence risk as part of our genetic counseling for this condition.

The lack of familial aggregation means that meiotic approaches to gene identification cannot be used. Instead, the gene will probably be identified by either a candidate gene approach, a patient with a recognizable chromosomal aberration and PS or whole genome assays that can distinguish subtle genomic differences in affected and unaffected tissues. We and several other groups are hard at work on these approaches and the affected families and we are eager for a breakthrough in this challenging and fascinating disorder of growth dysregulation.

\section{Acknowledgements}

I use first-person plural format in this paper to recognize the fact that the NIH Proteus syndrome research program is a team effort, including input from MM Cohen, Jr, Joyce Turner, Kathryn Peters, Julie Sapp, and a host of collaborators from many subspecialties, and the leaders of the US and UK Proteus syndrome foundations. Few of the ideas presented here are solely those of mine but are instead the product of many fruitful discussions with these committed and caring professionals and the affected families. I accept sole responsibility for any errors that may be present in this paper. The Proteus syndrome research project is supported by Intramural Funds from the NIH National Human Genome Research Institute. The opinions expressed here do not necessarily represent the policy or position of the NIH or any other institutions to which I am affiliated.

\section{References}

1 Cohen Jr MM, Hayden PW: A newly recognized hamartomatous syndrome. Birth Defects Orig Artic Ser 1979; 15: 291-296.

2 Wiedemann HR, Burgio GR, Aldenhoff P, Kunze J, Kaufmann HJ, Schirg E: The proteus syndrome. Partial gigantism of the hands and/or feet, nevi, hemihypertrophy, subcutaneous tumors, macrocephaly or other skull anomalies and possible accelerated growth and visceral affections. Eur J Pediatr 1983; 140: 5-12.

3 Cohen Jr MM: The elephant man did not have neurofibromatosis. Proc Greenwood Genet Center 1987; 6: 187-192.

4 Treves F: A case of congenital deformity. Proc Pathol Soc London 1885; 36: 494-498.

5 Biesecker LG: The multifaceted challenges of Proteus syndrome. JAMA 2001; 285: 2240-2243. 
6 Biesecker LG, Happle R, Mulliken JB et al: Proteus syndrome: diagnostic criteria, differential diagnosis, and patient evaluation. Am J Med Genet 1999; 84: 389-395.

7 Biesecker LG, Peters KF, Darling TN et al: Clinical differentiation between Proteus syndrome and hemihyperplasia: description of a distinct form of hemihyperplasia. Am J Med Genet 1998; 79: 311-318.

8 Turner JT, Cohen Jr MM, Biesecker LG: Reassessment of the Proteus syndrome literature: application of diagnostic criteria to published cases. Am J Med Genet 2004; 130A: 111-122.

9 Nguyen D, Turner JT, Olsen C, Biesecker LG, Darling TN: Cutaneous manifestations of proteus syndrome: correlations with general clinical severity. Arch Dermatol 2004; 140: 947-953.

10 Twede JV, Turner JT, Biesecker LG, Darling TN: Evolution of skin lesions in Proteus syndrome. I Am Acad Dermatol 2005; 52: 834-838.

11 Cohen Jr MM, Neri G, Weksberg R: Klippel-Treaunay syndrome, Parkes Weber Syndrome, and Sturge-Weber syndrome: Overgrowth syndromes. New York: Oxford University Press, 2002, pp 111-124.

12 Cohen Jr MM, Neri G, Weksberg R: Proteus syndrome: Overgrowth syndromes. New York: Oxford University Press, 2002, pp 75-110.

13 Wiedemann HR, Burgio GR: Encephalocraniocutaneous lipomatosis and Proteus syndrome. Am J Med Genet 1986; 25: 403-404.

14 Hotamisligil GS: Proteus syndrome and hamartoses with overgrowth. Dysmorphol Clin Genet 1990; 4: 87-107.

15 Darmstadt GL, Lane AT: Proteus syndrome. Pediatr Dermatol 1994; 11: $222-226$.

16 Biesecker LG, Rosenberg MJ, Vacha S, Turner JT, Cohen Jr MM: PTEN mutations and Proteus syndrome. Lancet 2001; 358: 2079-2080.

17 Smith JM, Kirk EP, Theodosopoulos G et al: Germline mutation of the tumour suppressor PTEN in Proteus syndrome. J Med Genet 2002; 39: 937-940.

18 Zhou XP, Marsh DJ, Hampel H, Mulliken JB, Gimm O, Eng C: Germline and germline mosaic PTEN mutations associated with a
Proteus-like syndrome of hemihypertrophy, lower limb asymmetry, arteriovenous malformations and lipomatosis. Hum Mol Genet 2000; 9: 765-768.

19 DeBaun MR, Brown M, Kessler L: Screening for Wilms' tumor in children with high-risk congenital syndromes: considerations for an intervention trial. Med Pediatr Oncol 1996; 27: 415-421.

20 Zhou X, Hampel $\mathrm{H}$, Thiele $\mathrm{H}$ et al: Association of germline mutation in the PTEN tumour suppressor gene and Proteus and Proteus-like syndromes. Lancet 2001; 358: 210-211.

21 Cohen Jr MM: Proteus syndrome: an update. Am J Med Genet C Semin Med Genet 2005; 137: 38-52.

22 Cohen MMJ, Turner JT, Biesecker LG: Reply to Kirk, et al. Am J Med Genet 2004; 130A: 216-217.

23 Cohen Jr MM, Turner JT, Biesecker LG: Proteus syndrome: misdiagnosis with PTEN mutations. Am J Med Genet 2003; 122A: $323-324$.

24 Barker K, Martinez A, Wang R et al: PTEN mutations are uncommon in Proteus syndrome. J Med Genet 2001; 38: 480-481.

25 Robin NH, Biesecker LG: Considerations for a multiaxis nomenclature system for medical genetics. Genet Med 2001; 3: 290-293.

26 Biesecker LG: Proteus syndrome; in Cassidy SB, Allanson J (eds): Management of Genetic Syndromes. Hoboken: John Wiley and Sons, Inc., 2005, vol 1, pp 449-456.

27 Cohen Jr MM: Causes of premature death in Proteus syndrome. Am J Med Genet 2001; 101: 1-3.

28 Slavotinek AM, Vacha SJ, Peters KF, Biesecker LG: Sudden death caused by pulmonary thromboembolism in Proteus syndrome. Clin Genet 2000; 58: 386-389.

29 Gordon PL, Wilroy RS, Lasater OE, Cohen Jr MM: Neoplasms in Proteus syndrome. Am J Med Genet 1995; 57: 74-78.

30 Happle R: Lethal genes surviving by mosaicism: a possible explanation for sporadic birth defects involving the skin. J Am Acad Dermatol 1987; 16: 899-906.

31 Happle R: Cutaneous manifestation of lethal genes. Hum Genet 1986; 72: 280. 